\title{
Ceramic Coatings for High-Tech Applications
}

\author{
For a long time, the production of ceramic coatings has only been possible by means of \\ sintering techniques conducted at more than $1,000{ }^{\circ} \mathrm{C}$. However, a novel spraying \\ method, Powder Aerosol Deposition (PAD), enables their production at normal room \\ temperatures. It is therefore highly attractive for industrial applications. Engineering scientists \\ from the University of Bayreuth, Germany, are working in the frontline of ongoing \\ development of this technology.
}

With PAD, dense ceramic films can be applied to very different types of materials, such as steel, glass, silicon, or even plastic. To achieve this, a dry ceramic powder is first converted into an aerosol, which is a mixture of gas and solid particles, with the aid of a carrier gas. The aerosol is then transported into a vacuum chamber, and accelerated to several 100 meters per second through a nozzle and directed onto the material to be coated. On impact, the tiny ceramic particles fracture. The resulting fragments, only a few nanometers in size, feature fresh, active surfaces. They form tightly adhering, dense coatings with a thickness of between 1 and $100 \mu \mathrm{m}$ (Figure 1). "Thanks to their dense microstructure, the coatings already exhibit excellent mechanical properties even directly after the deposition. They are extraordinarily hard and have good chemical resistance," explains Dr.-Ing. Jörg Exner, first author of the study, who was a driving force in the research work on PAD at the University.

However, as it turned out, some functional properties of the coatings, especially the electrical conductivity, proved inadequate without carrying out further steps. In their new study, nevertheless, the Bayreuth engineering scientists are now able to report on effective methods of optimization.

Crystalline structures are of crucial importance in this context. The strong impact of the ceramic particles on the materials causes structural defects in the resulting fragments. This not only affects electrical conductivity, but also other functional properties. "By a thermal post-treatment, or so-called tempering, these defects can be almost com-

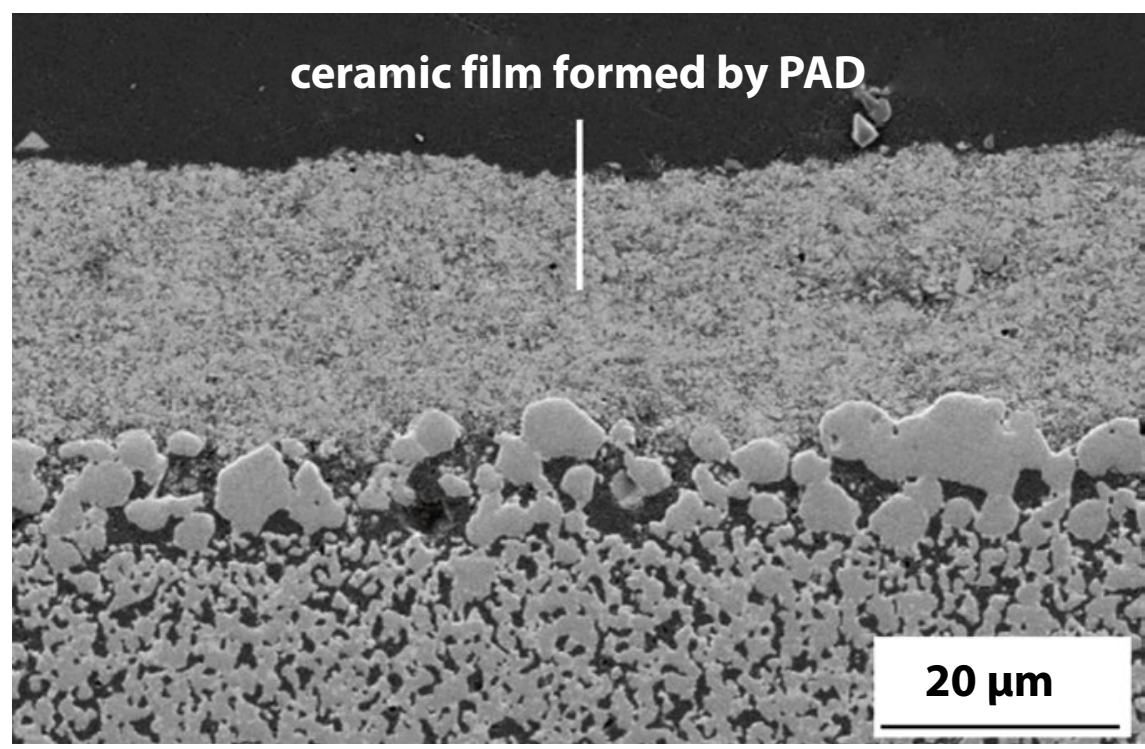

Figure 1 A ceramic film produced by powder aerosol deposition on a porous gas-permeable electrode, such as those required in fuel cells. (@ Jörg Exner)

pletely eliminated. We have been able to show that the required temperatures are generally much lower than for conventional sintering. The avoidance of these extremely high temperatures is what makes PAD so attractive. It therefore remains true: This technology offers very high industrial potential, especially where high-quality ceramic coatings are required," Exner concludes.

What type of ceramic materials are processed depends on the intended technological applications: Dielectric ceramics are suitable for capacitors, electrically conductive functional ceramics are preferred for sensors, and yttrium-stabilized zirconium oxide is used in high-temperature fuel cells. Even lithium-ion batteries can be produced in this way.
The scientific understanding of the ceramic film structures and of their functional properties, gained at the University of Bayreuth, will contribute significantly to the goal of integrating high-quality coated components into complex systems in a sustainable way. New technologies, for example, in the fields of energy storage and conversion, or for the purpose of environmental monitoring, therefore stand to benefit considerably from powder aerosol deposition applications.

Contact:

Functional Materials, University of Bayreuth, Bayreuth, Germany, www.cme-keramik.uni-bayreuth.de/en 